Proceedings of the 1999 Particle Accelerator Conference, New York, 1999

\title{
REALISTIC PREDICTION OF DYNAMIC APERTURE AND OPTICS PERFORMANCE FOR LEP
}

\author{
John M. Jowett*, CERN, Geneva, Switzerland
}

\begin{abstract}
Over the two-decade lifetime of the LEP project, techniques for evaluating the quality of optical configurations have evolved considerably to exploit the growth in computer power and improved modelling of single-particle dynamics. These developments have culminated in a highly automated Monte-Carlo evaluation process whose stages include the generation of an ensemble of imperfect machines, simulation of the operational correction procedures, correlation studies of the optical functions and parameters of (both) beams, 4-dimensional dynamic aperture scans and tracking with quantum fluctuations to determine the beam core distribution. We outline the process, with examples, and explain why each step is necessary to realistically capture essential physics affecting performance. The mechanisms determining the vertical emittance, radial beam distribution and dynamic aperture are especially important. As a storage ring in which an unusual variety of optics have been tested, LEP provides a valuable test case for the predictive power of the methodology.
\end{abstract}

\section{INTRODUCTION}

Persistent disagreement of, say, a factor of two between computed and measured dynamic apertures would invalidate computation as a rational means of assessing the quality of a storage ring optics.

However, the potential of an optical configuration only becomes clear after some weeks of operational performance maximisation (and may also require hardware changes). Such tests are expensive in large machines like LEP. Accurate computational appraisal is therefore vital to estimate not only dynamic aperture but also-and consistently - the gamut of optical quantities culminating in the beam sizes at the collision points.

The conceptual framework and tools brought to bear on the problem of performance prediction for LEP have evolved considerably over some 20 years. Rather than review the history (traceable through the citations), this paper will describe current best efforts to model the various LEP optics, emphasising recent high energy operation (LEP2) where the synchrotron radiation effects are strong.

\section{CONCEPTS AND METHODOLOGY}

In this paper, computing the linear machine is shorthand for the calculation of the $6 \mathrm{D}$ closed orbit including radiation, the eigenvectors of linear oscillations around it (hence all optical functions and the canonical transformations to

*John.Jowett@ cern.ch, http://wwwslap.cern.ch/ jowett/ the eigenmodes), the 3 tunes, energy loss, damping rates, emittances, etc. Usually, the eigenmodes correspond approximately to $(1,2)$ horizontal and vertical "betatron" oscillations and (3) "synchrotron" oscillations. These calculations are implemented in the program MAD [1].

In MAD's tracking, radiation damping and quantum excitation arise naturally [2] because all particle momentum changes are computed locally, according to the distribution of $\gtrsim 300 \mathrm{RF}$ cavities and the canonical co-ordinates of the particle in each multipole magnet.

The dynamic aperture is the basin of attraction of the closed orbit in deterministic tracking with radiation damping [2] (without quantum fluctuations). This definition is computationally unambiguous and independent of the number of turns tracked beyond a certain minimum (for LEP2, 100 turns are ample).

The dynamic aperture of LEP is essentially determined by three classes of intentional non-linear elements: chromaticity sextupoles, RF cavities and focusing quadrupoles. Although quadrupoles provide linear focusing, their nonlinear radiation terms are responsible for the ultimate dynamic aperture limit. (Higher order multipole errors are less important.)

A dynamic aperture scan is carried out by varying the action variables $\left(I_{1}, I_{2}, I_{3}\right) \in\left(\mathbb{R}^{+}\right)^{3}$ over a spherical polar grid $\mathbb{R}^{+} \times[0, \pi / 2]^{2}$. At each point, the phase $\phi_{3} \in[0,2 \pi)$ of the third mode is also scanned. A dynamic aperture is typically the result of tracking 1000-2000 particles and is returned as a surface in the 3D action space [3].

The correction procedures applied are designed to етиlate the real operational procedures, as responses to measured quantities without knowledge of the imperfections, rather than the best that could be done computationally.

In outline, the optics evaluation procedure is:

1. Generate ideal machine with detector solenoids, RF, and radiation switched off.

2. Install vacuum chamber elements in all quadrupoles to provide aperture limitations in tracking.

3. Compute solenoid compensation with tilted quadrupoles, to be applied in calculations with the solenoids on.

4. Compute linear machine and dynamic aperture scan of ideal machine (with solenoids, RF and radiation on) as a reference.

5. Generate an ensemble (typically 30 ) of machines with typical random misalignments, tilts and field errors in all dipoles, quadrupoles and sextupoles.

For each machine in the ensemble,

(a) Correct the non-radiating closed orbit independently in the two planes (to $\sqrt{\left\langle x^{2}\right\rangle}=0.6 \mathrm{~mm}$, 

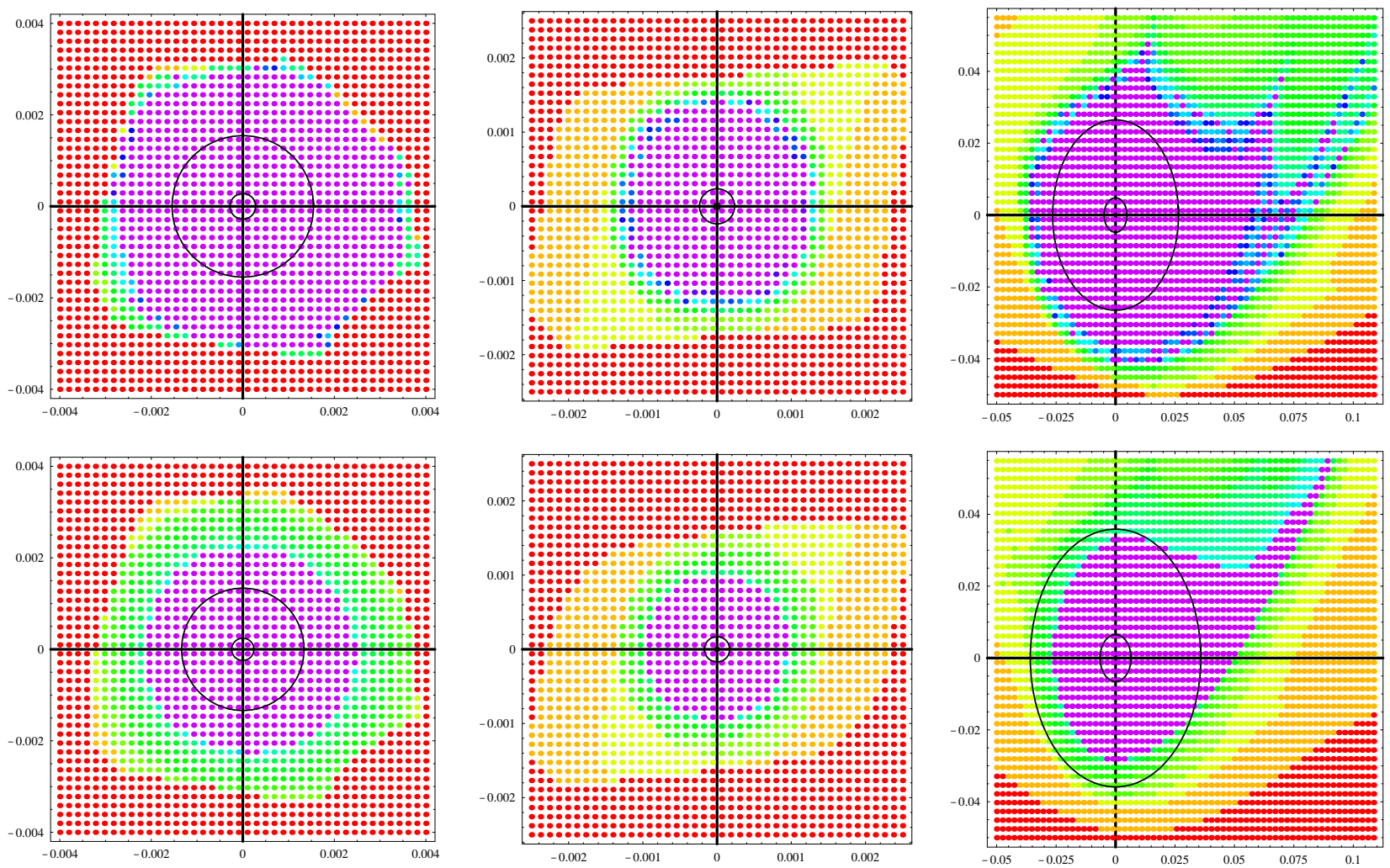

Figure 1: Shrinking of the dynamic aperture at the ultimate energy: the upper row shows survival plots in the normalised phase planes (all co-ordinates in $\sqrt{\mathrm{m}}$ ) of the 3 normal modes at $94 \mathrm{GeV}$ with $J_{x}=1$ and a comfortable $V_{\mathrm{RF}}=2961 \mathrm{MV}$. The lower row shows the same imperfect machine at $100 \mathrm{GeV}$ with $J_{x}=1.5$ and $V_{\mathrm{RF}}=3265 \mathrm{MV}$ just sufficient. Contours of 1 and $5.5 \sigma$ of the notional gaussian distributions are shown to give the scale. Most of these 6400 particles rotate anti-clockwise from the initial conditions shown. Initial conditions are coloured with a hue indicating survival time, ranging from violet ( dark central stable zone) to red (loss in one turn).

$\left.\sqrt{\left\langle y^{2}\right\rangle}=0.4 \mathrm{~mm}\right)$.

(b) Switch on solenoids, RF and radiation. For the $\mathrm{e}^{+}$beam, correct the IP optics $\left(\beta_{y}^{*}\right)$ and tunes using "knobs" as in operation.

(c) Save all the imperfections and their corrections, reverse the ideal machine structure and rebuild the physically equivalent machine for the $\mathrm{e}^{-}$ beam. Thus, some corrections appropriate for the positrons are applied to the electron beam and may enhance the differences between the beams. Tune splits between beams can be $\gtrsim$ 0.01 , depending on the distribution of $V_{\mathrm{RF}}$.

(d) Compute the linear machine and carry out a dynamic aperture scan for each beam.

(e) Track with quantum fluctuations [2] (for $\gtrsim 200$ ) damping times) to estimate non-linear effects on the emittances and beam sizes.

This process involves $\gtrsim 1000$ multifarious runs of MAD, all managed by a Mathematica [4] notebook interface that prepares the input and absorbs the results into a structured database of function definitions.

Other notebooks load the database to display and correlate machine parameters or generate comprehensive re- ports [6] on an optics. These include orbit and optics at key elements, emittances, tunes, survival data and dynamic apertures and differences between beams. The environment is open-ended and congenial: it is easy to compute derived quantities such as beam-overlaps or centre-of-mass energies at the experiments; several databases can be loaded to make comparisons across optics, etc.

\section{SAMPLE RESULTS}

\subsection{Dynamic aperture}

LEP has operated with several optical configurations $[3,7]$ with varying arc cell phase advances $\left(\mu_{x}, \mu_{y}\right)$. Dynamic aperture measurements have been made, where possible, with two or three different methods.

For certain optics, results of tracking with radiation are quantitatively in agreement with earlier calculations without radiation. This is the hallmark of certain physical mechanisms, e.g., detuning with amplitude onto an integer resonance. Including radiation, or even imperfections, makes little quantitative difference although the radiative beta-synchrotron coupling instability (RBSC) [2, 3] effect always steps in to accelerate initial amplitude growth. In 

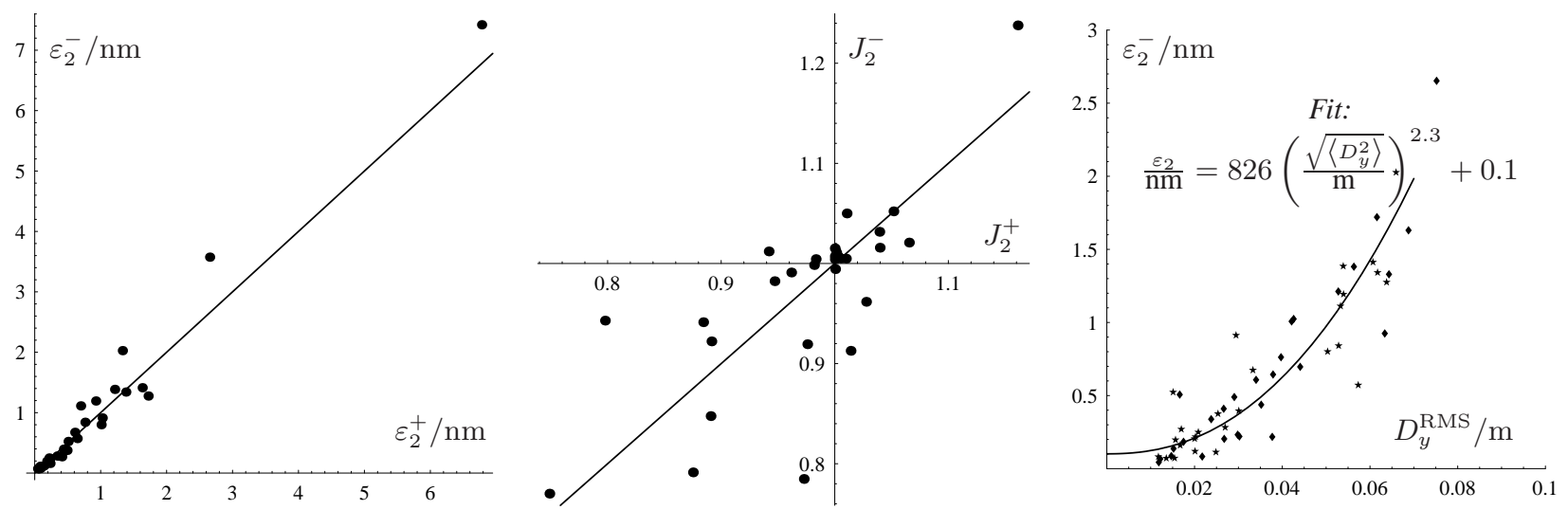

Figure 2: Correlation of vertical emittance and damping partition numbers between $\mathrm{e}^{+} \mathrm{e}^{-}$; correlation of vertical emittance (both beams) and RMS vertical dispersion, $D_{y}^{\mathrm{RMS}}$. The fit tests the hypothesis $\varepsilon_{2} \simeq a\left(D_{y}^{\mathrm{RMS}}\right)^{2} / J_{2}+\varepsilon_{c}=a\left(D_{y}^{\mathrm{RMS}}\right)^{p}+\varepsilon_{c}$.

other cases, e.g., higher order resonances or pure RBSC, radiation and imperfections are important, the dynamic aperture has a spread and is less than for an ideal machine. Radiation effects are essential in all cases to determine the stability limit of mode 3 ("momentum acceptance").

There is no space to discuss measurements here. However a recent survey $[8,5]$ showed that calculation and measurement agree within about $10 \%$ in all cases where satisfactory measurements have been made.

Figure 1 has been computed for the present optics with $\left(\mu_{x}, \mu_{y}\right)=\left(102^{\circ}, 90^{\circ}\right)$ to show the effect of moving from a situation with a reserve of RF voltage to "ultimate" LEP parameters at $100 \mathrm{GeV}$ where $V_{\mathrm{RF}}$ is just sufficient according to the conventional quantum lifetime calculation. All details of this figure can be understood by analysing the orbits of individual particles. At $94 \mathrm{GeV}$, the stability limit of mode 1 is determined by the cross-detuning effect: $Q_{2}$ moves down to the integer resonance with increasing $I_{1}$. The boundary between stability and loss in one turn is sharp. At $100 \mathrm{GeV}$, the transverse dynamic apertures are sharply reduced because the limit is now determined by the RBSC instability. Since the growth time of this instability is $\simeq 2 / Q_{3} \simeq 20$ turns, a "ghost" of the former dynamic aperture remains visible. Similar effects are visible in mode 2 which was already limited by RBSC.

In mode 3 , the dynamic aperture becomes simpler in shape although this example shows why a scan of $\phi_{3}$ is essential. It is clear that the approximations underlying existing analytic approaches to the calculation of quantum lifetime are inadequate in this regime.

\subsection{Vertical emittance}

The emittance of mode $2, \varepsilon_{2}$, is a crucial parameter determining luminosity. It is determined almost entirely by machine imperfections and so can only be estimated statistically. Figure 2 shows predictions for the same optics at $94 \mathrm{GeV}$. A mismatch of $\varepsilon_{2}$ between beams can arise, partly because the damping partition numbers $J_{2}$ can be different. Fitting shows that $\varepsilon_{2}$ is determined mainly by the RMS ver- tical dispersion. The dependence is stronger than quadratic because $J_{2}$ tends to decrease with the dispersion (because of combined dispersion and closed orbit in quadrupoles).

The statistical distribution of vertical emittance in the ensemble is typical of measurements during the life-cycle of an optics. Operational procedures seem to select corrections that reduce $\varepsilon_{2}$ to the smallest values.

Synchro-betatron resonances can increase $\varepsilon_{2}$ further. Such effects can be estimated by tracking particles with quantum fluctuations [5].

\section{CONCLUSIONS}

By carefully constructing ensembles of model machines, simulating the operational correction procedures and using a physically faithful model of single particle dynamics, it is possible to predict the distribution of beam parameters and their differences between the beams in LEP. Dynamic aperture can be predicted to within about $10 \%$ in a variety of optical configurations.

At the highest accessible energies, the dynamic aperture of LEP will be sharply reduced by the RBSC instability.

Acknowledgements: I thank A. Verdier for valuable collaboration, K. Goral for help with software development.

\section{REFERENCES}

[1] F.C. Iselin, http://wwwslap.cern.ch/ $\sim$ fci/mad/mad_home.html

[2] F. Barbarin, et al, Proc. 4th European Particle Accelerator Conference (EPAC'94), World Scientific, p. 193.

[3] Y. Alexahin et al, EPAC'96, Sitges, IOP Publishing, 1996.

[4] S. Wolfram, Mathematica, Cambridge Univ. Press, 1996.

[5] J.M. Jowett, Proc. 8th LEP Performance Workshop, CERN SL/98-006 (1998).

[6] J.M. Jowett, CERN-SL Notes 97-84, 98-020, 98-072 (199798). See also [5].

[7] D. Brandt et al EPAC'98, Stockholm, IOP Publishing, 1998.

[8] H. Burkhardt, Proc. 8th Workshop on LEP Performance, CERN-SL-98-006 (1998). 